\title{
Undifferentiated carcinoma of the oral tongue treated with cetuximab: A case report
}

\author{
YUKI SAKAMOTO, SOUICHI YANAMOTO, MASAKI ADACHI, SHOMA TSUDA, KOHEI FURUKAWA, \\ TOMOFUMI NARUSE and MASAHIRO UMEDA
}

Department of Clinical Oral Oncology, Graduate School of Biomedical Sciences, Nagasaki University, Nagasaki 852-8588, Japan

Received July 25, 2018; Accepted January 30, 2019

DOI: $10.3892 /$ mco.2019.1808

\begin{abstract}
Undifferentiated carcinoma of the oral cavity is a rare and possibly fatal malignant neoplasm. We present here a case of undifferentiated carcinoma of the oral tongue, which responded well to chemotherapy with cisplatin, 5-fluorouracil, and cetuximab. A 59-year-old man was referred to our hospital for a large tumor of the tongue. Physical examination revealed an ulcerative tumor, approximately $50 \mathrm{~mm}$ in diameter, on the right edge of the tongue and swelling of multiple cervical lymph nodes; therefore, the clinical diagnosis was tongue cancer (T4aN2bM0). The histological diagnosis from a biopsy specimen was undifferentiated carcinoma; we tested the sample for human papilloma virus immunoreactivity, which was positive. The patient then underwent chemotherapy with cisplatin, 5-fluorouracil and cetuximab. The tumor's size decreased markedly after two courses of chemotherapy, but the treatment was stopped due to the patient' various mental problems and pneumonia; he died 6 months after the initial visit. Our encounter with this patient suggests that cetuximab treatment is a good option for HPV-positive undifferentiated carcinoma of the oral tongue.
\end{abstract}

\section{Introduction}

Although undifferentiated carcinomas are common in the nasopharynx, reports of occurrences in the oral cavity are extremely rare (1-3). In particular, there have been no reports of undifferentiated carcinoma that originated in the oral

Correspondence to: Dr Souichi Yanamoto, Department of Clinical Oral Oncology, Graduate School of Biomedical Sciences, Nagasaki University, 1-7-1 Sakamoto, Nagasaki 852-8588, Japan

E-mail: syana@nagasaki-u.ac.jp

Abbreviations: 5FU, 5-fluorouracil; CT, computed tomography; PET, positron emission tomography; HPV, human papillomavirus; EBV, Epstein-Barr virus

Key words: undifferentiated carcinoma, oral tongue, cetuximab, cisplatin, 5-fluorouracil, human papilloma virus portion of the tongue. Undifferentiated carcinomas related to Epstein-Barr virus (EBV) and human papillomavirus (HPV) have been reported $(1,4,5)$. Head and neck squamous cell carcinomas related to EBV or HPV are generally more sensitive to chemotherapy and have better prognoses (5-7); however; chemotherapeutic efficacy is unclear with respect to undifferentiated carcinoma of the oral cavity. Cetuximab, a molecular agent targeted against epidermal growth factor receptor, is reported to have significant efficiency in treatment of head and neck squamous cell carcinoma (8). We present here a case of locally advanced, undifferentiated carcinoma of the oral tongue that responded well to chemotherapy with cisplatin, 5-fluorouracil (5-FU) and cetuximab. This is the first case of undifferentiated carcinoma of the oral tongue, and the first report of the use of cetuximab to treat undifferentiated carcinoma in the head and neck.

\section{Case report}

A 59-year-old Japanese man was referred to our hospital in April 2016, because of a painless nodule on the right-side of his tongue. He had a history of hepatitis B and alcohol-related psychosis; moreover, he had been admitted three times to a psychiatric hospital for a mental disorder. His nutrition was poor, because eating was difficult as the tumor grew. Physical examination showed an elastic, hard, tumorous lesion on the edge of the tongue, with a size of approximately $45 \times 50 \mathrm{~mm}$ (Fig. 1). The overlying mucosa was an almost smooth surface and focal erosion. There were multiple, enlarged cervical lymph nodes in the upper- and mid-jugular regions.

Computed tomography (CT) revealed a large tumor diffusely invading into the sublingual space, anterior edge of the hyoid bone, genioglossus, and mylohyoid muscle (Fig. 2A). The cervical lymph nodes near the internal jugular veins were uniformly enlarged and were impinging on the internal jugular vein and common carotid artery (Fig. 2B). Distant metastasis or any other primary tumor was not observed by positron emission tomography (PET)-CT.

Under a clinical diagnosis of tongue cancer (T4aN2bM0), incisional biopsy was performed. The specimen was a tumor sample consisting of diffuse, large, atypical cells in the submucosa and muscle. The cell nuclei were variously sized and were atypical, hyperchromatic and multinucleated. There were also 


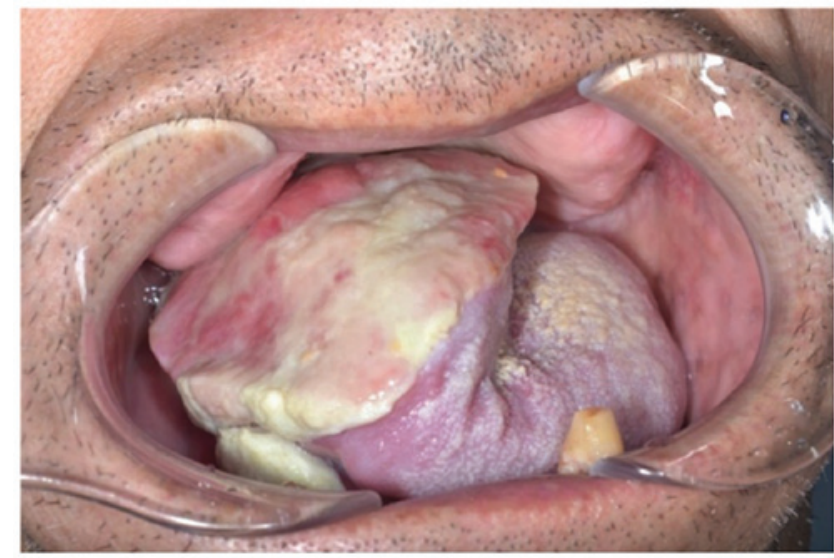

Figure 1. Intraoral appearance. Physical examination revealed a pink-white, smooth appearance with an ulceration.
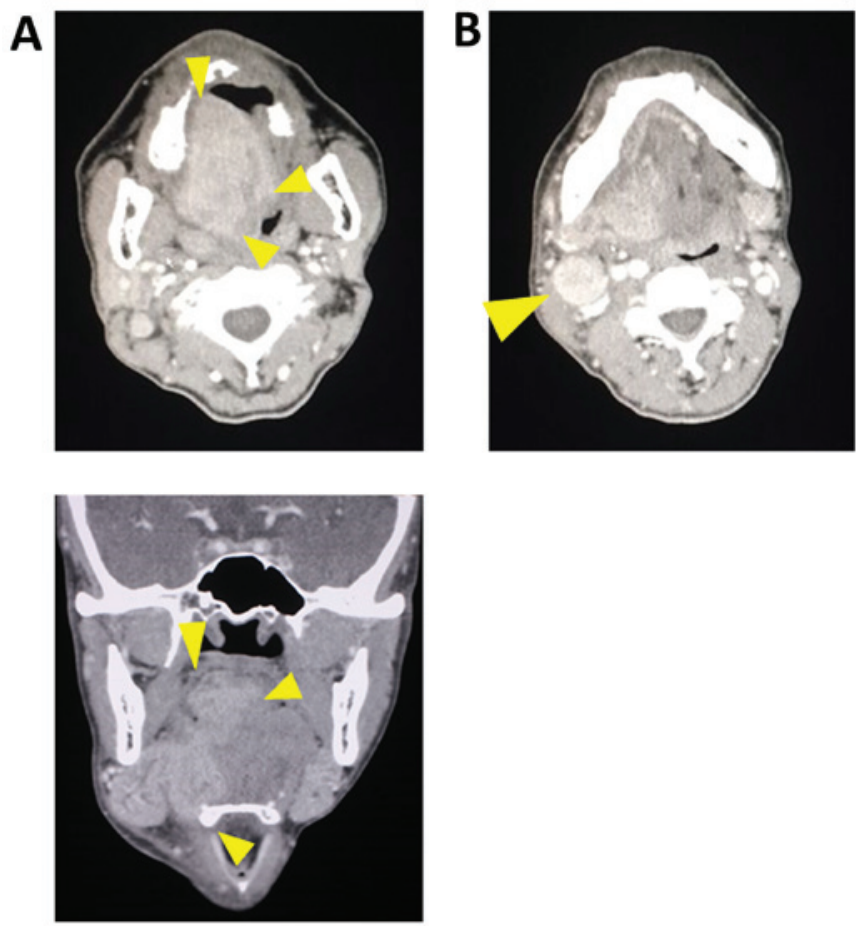

Figure 2. Computed tomography imaging. (A) Large tumor diffusely invading into the sublingual space, anterior edge of the hyoid bone, genioglossus, and mylohyoid muscle (arrowheads). (B) Uniformly enlarged cervical lymph node impinging on the internal jugular vein and common carotid artery (arrowheads).

many instances of nuclear fissions, polygon-shaped cells, and anaplastic cells (Fig. 3). Immunohistochemistry revealed positive labeling for a marker of epithelial cells (cytokeratin AE1/AE3; Fig. 4) and negative labeling for markers of lymphocytes and blood cells (CD30, CD20, CD68, UCHL-1L26, CD79a, Granzyme B, TIA-1, ALK and EBV-LMP) and muscle and nerve cells (desmin and S-100B). Based on these findings, the tumor was diagnosed as undifferentiated carcinoma of the tongue. To evaluate the relationship between undifferentiated carcinomas and HPV, we tested the sample for p16 immunoreactivity (p16INK4A), which was positive (Fig. 5A and B).

We initially recommended surgery to the patient and his family but did not obtain consent, because of the possibility of
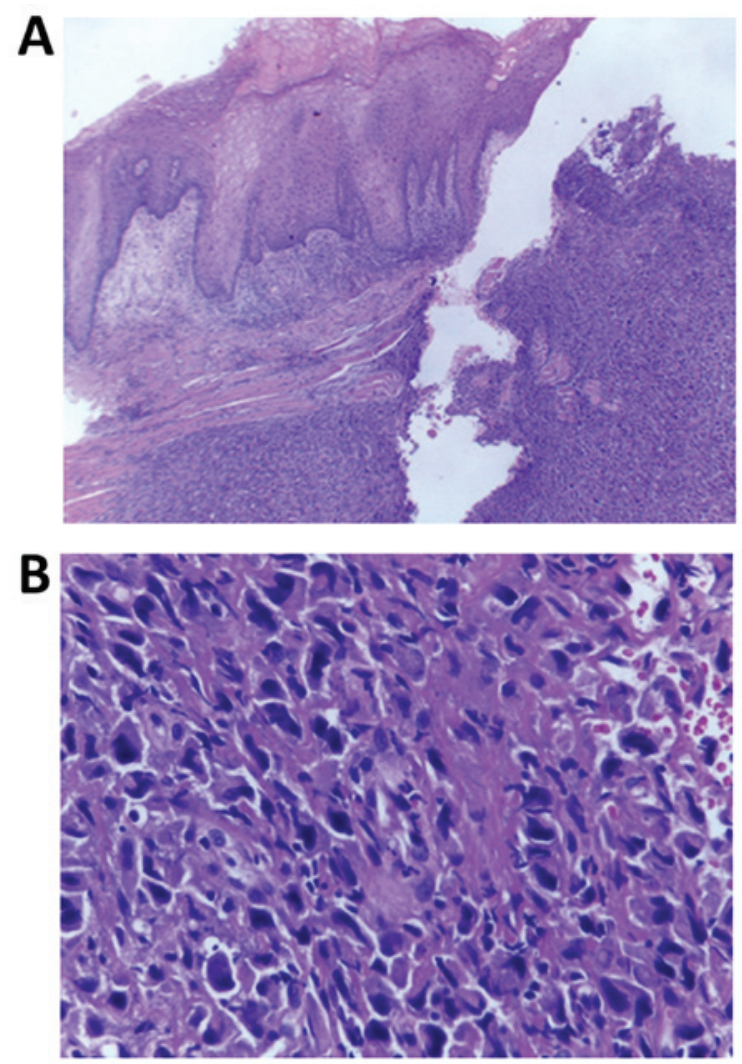

Figure 3. Representative histopathological features. Hematoxylin and eosin (H\&E) staining. (A) Mucosa with ulceration. The continuity of the basement membrane is maintained (x10). (B) The number of diffuse, large, atypical cells are increased in the submucosa and muscle. The cell nuclei are variously sized, and cells are atypical, hyperchromatic, and multinucleated. There are many instances of nuclear fissions, polygon-shaped cells and anaplastic cells (x200).

functional issues, such as eating and speaking, after surgery, as well as in the context of his mental disorder. Therefore, chemotherapy with cisplatin, 5-FU and cetuximab was selected instead as NAC therapy. When the tumor shrank, we planned to arrange surgery. The patient received cetuximab (1st course: $400 \mathrm{mg} / \mathrm{m}^{2}$; 2nd course: $250 \mathrm{mg} / \mathrm{m}^{2}$ ) every week and cisplatin $\left(100 \mathrm{mg} / \mathrm{m}^{2}\right)$ and 5 -FU $\left(1,000 \mathrm{mg} / \mathrm{m}^{2}\right)$ every 3 weeks. No severe adverse events occurred. Leukopenia was observed with a white blood cell count of 2,500 cells $/ \mathrm{mm}^{3}$, and drug eruption was Grade 1. After two of these treatment cycles, the patient developed pneumonia, and chemotherapy was stopped. The intraoral tumor showed complete response, and the metastatic lymph nodes in the neck decreased in size (Fig. 6A-D).

Despite these improvements, the patient ate against medical advice, refused antibiotic administration, acted violently against the nurses, and his mental disorder worsened. We repeatedly consulted his family and the psychiatrist; however, his behavior and mental condition did not improve; thus, all treatment was stopped. After the patient recovered from pneumonia, he was transferred to a hospital that specialized in palliative care. Three months after the last course of chemotherapy, he underwent palliative radiotherapy (40 Gy) due to the rapid spread of metastasis in the neck. There was no evidence of a recurrent tumor in the oral cavity, but he died from the metastatic tumor in his neck 2 months after the end 


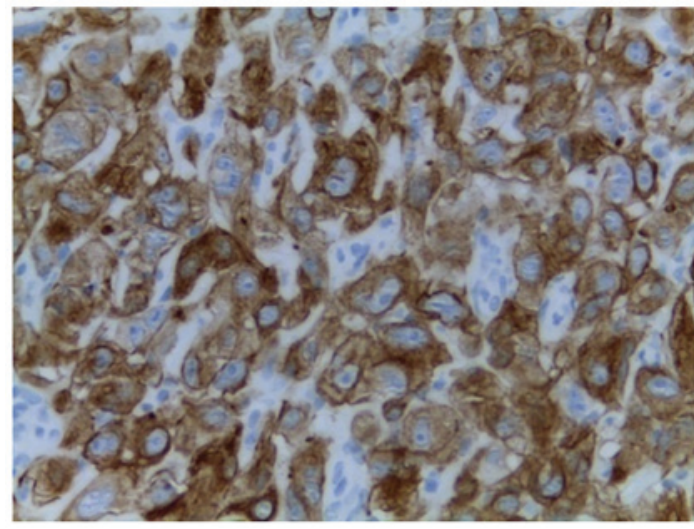

Figure 4. Immunohistochemical examination of tumor biopsy specimens shows strong labeling for cytokeratin AE1/AE3 (x400).

of radiotherapy. It was not clear whether distant metastases had developed, because examinations for metastases were not performed during the period of palliative care.

\section{Discussion}

Undifferentiated carcinoma comprises $0.2 \%$ of all malignant tumors of the head and neck and has an extremely poor prognosis $(4,9)$. However, the occurrence of undifferentiated cancer in the oral cavity is extremely rare; notably, several cases have been reported in the salivary glands $(2,3)$. To the best of our knowledge, this is the first case of undifferentiated carcinoma of the oral tongue.

Initially, we had assumed that the patient's tumor was an anaplastic carcinoma. Therefore, immunohistochemistry was performed, both for diagnosis and to choose the best treatment plan. Diagnosis of undifferentiated carcinoma is difficult, but immunohistochemical labeling is useful. It shows positive reactivity for the epithelial marker cytokeratin AE1/AE3 and negative reactivity for $\mathrm{S}-100$, hematolymphoid markers, and melanocytic cell markers $(5,10,11)$. Our findings were consistent

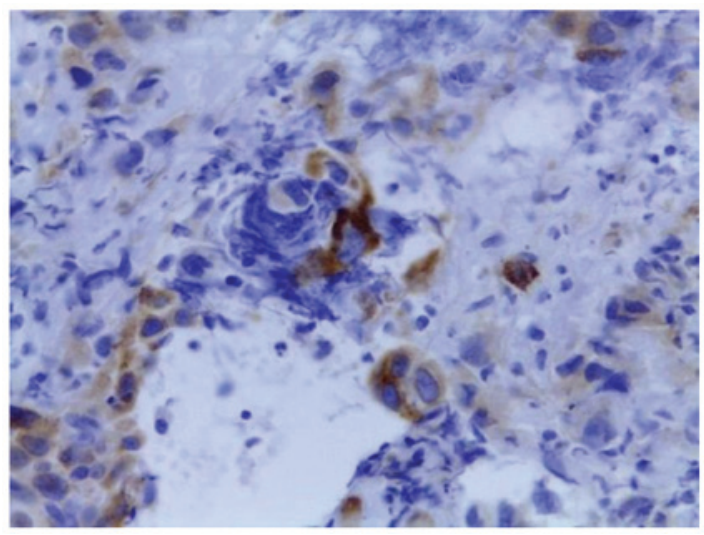

Figure 5. Immunohistochemical examination of tumor biopsy specimens shows positive staining for p16 in the cytoplasm of tumor cells (x400).

with these diagnostic criteria. Although we elected to treat with chemotherapy alone, chemotherapy and radiation might have been a better choice based on the immunohistochemical findings.

Undifferentiated carcinomas related to Epstein-Barr virus (EBV) and HPV have been reported $(1,4,5)$. EBV disrupts normal cell division by making EBV-positive cells cancerous and increasing the number of infected genomes (12-14). In the current case, the relationship with undifferentiated carcinoma and EBV infection could not be demonstrated, because the immunoreaction of EBV-LMP was negative. Notably, approximately $33.1 \%$ of healthy people are infected with HPV (15). There are various types of HPV, and a genotype is defined as positivity of the p16 immunoreactivity if any type of HPV is present. Furthermore, HPV has a high affinity for the epithelium; HPV is found in epithelial cells in 2.9 to $80.4 \%$ of head and neck cancers (15) and is frequently found in poorly differentiated squamous cell carcinoma (16). HPV infection rates in benign and malignant tumors in the oral cavity are reported to be between 20 and $85 \%$; this range is thought to be due to differences in HPV detection and sampling
A
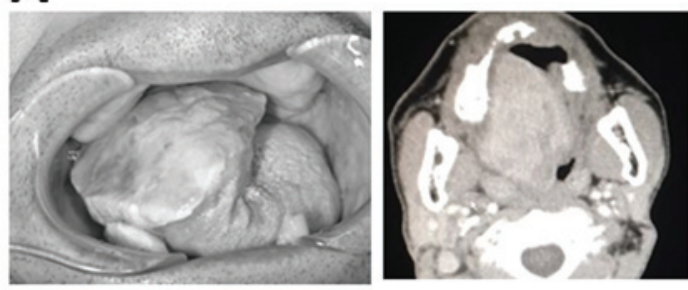

C
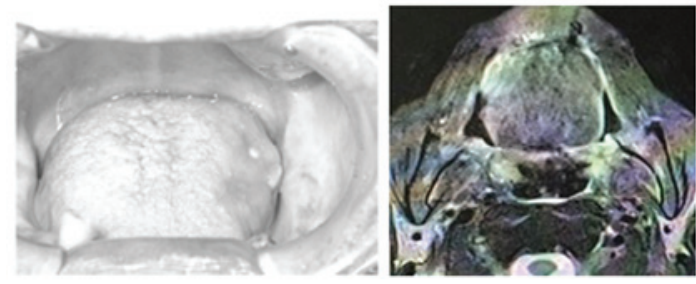

B

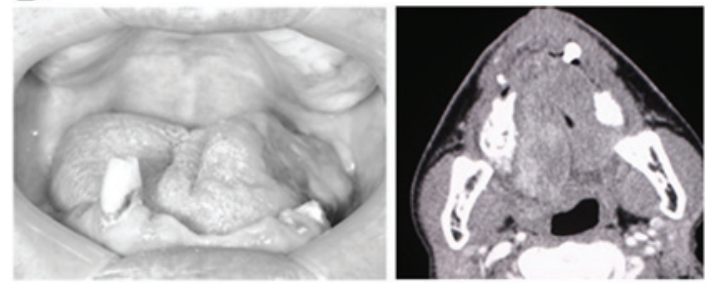

D

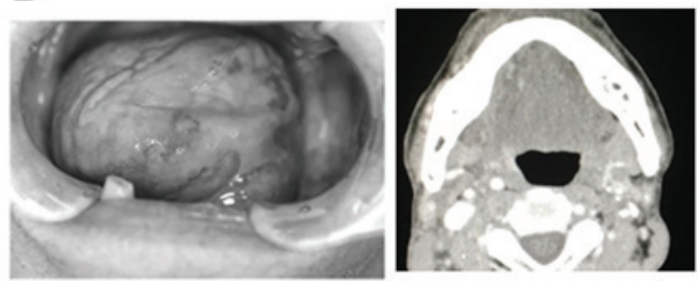

Figure 6. Representative clinical results and CT/MR images. (A) First visit. (B) After the 2nd course of chemotherapy. (C) Upon discharge from the hospital. (D) After palliative radiation. 
methods $(15,16)$. In general, HPV-positive patients are known to have better prognosis than HPV-negative patients $(1,4,5,7)$. HPV-positive cases of head and neck cancer are sensitive to chemotherapy and have been reported to have good prognoses $(1,7,17)$. Chemoradiotherapy is generally the first choice for HPV-positive oropharyngeal cancer. However, in head and neck cancer, if the tumor can be resected, surgical treatment is the first choice. Therefore, chemotherapy is not the first choice in the field of oral cancer. In this case, the aim of treatment was shrinking the tumor as NAC therapy; if the tumor had responded to treatment, we would have planned surgery. HPV has good compatibility with the epithelium. The mechanism of cetuximab inhibits cancer cell proliferation by binding to epidermal growth factor receptor. Therefore, we selected cetuximab as preoperative chemotherapy. In general, TPF is used for preoperative chemotherapy for head and neck cancers; however, we changed this to cetuximab as we suspected that TPF might have been too strong for this patient. The phase III EXTREME trial reported that the addition of cetuximab to platinum/5-FU significantly improved OS, compared with platinum/5-FU in head and neck squamous cell carcinoma (8). However, Vermorken et al subsequently examined this phase III EXTREME trial retrospectively and reported that the additional effect of cetuximab was unrelated to the status of HPV infection (17). Because HPV-positive cases are sensitive to chemotherapy, it seems that it was not possible to obtain a sufficient effect of cetuximab to produce a statistically significant difference. Bonner et al concluded that HPV infection is a prognostic biomarker of head and neck squamous cell carcinoma, but not predictive of response to cetuximab treatment (18). Therefore, they concluded that the benefits of cetuximab were obtained in both HPV-positive and negative cases. In our case, a positive reaction for p16 in a biopsy specimen was obtained by immunohistochemical examination, upon the addition of cetuximab to platinum/5-FU, the so-called EXTREME regimen, suggesting improved outcome. In this case, because of the primary tumor size reduction and no appearance of new cancer lesion, we evaluated that the chemotherapy was effective. However, HPV positive head and neck cancer is susceptible to chemotherapy and reported as having a good prognosis, we should have decided the chemotherapy with emphasis on side effects of chemotherapy.

The treatment plan and outcome for the patient described in this report was greatly hampered by the patient's mental and behavioral conditions. However, a satisfactory clinical course could have been obtained by accurately diagnosing the undifferentiated carcinoma. This patient selected chemotherapy as a NAC treatment. If his condition had been good and the tumor could have been resected, prognosis could have been good. Therefore, we suggest that cetuximab treatment is a one of the option for HPV-positive undifferentiated carcinoma of the oral tongue.

\section{Acknowledgements}

Not applicable.

\section{Funding}

No funding was received.

\section{Availability of data and materials}

The datasets used during the current study are available from the corresponding author on reasonable request.

\section{Authors' contributions}

YS and MU performed the case study, collected the data and images of the case and produced the draft of the manuscript. MA, TN, ST and KF critically analyzed the manuscript for important intellectual content. YS and SY analyzed patient data and wrote the manuscript. All authors read and approved the final manuscript.

\section{Ethics approval and consent to participate}

This study was granted an exemption from requiring ethics approval, the name of the ethics committee is Nagasaki University Hospital and the exemption number is 18082041.

\section{Patient consent for publication}

Releases of the present case were obtained from the patient's family.

\section{Competing interests}

The authors declare that they have no competing interests', and all authors confirm its accuracy.

\section{References}

1. Carpenter DH, El-Mofty SK and Lewis JS Jr: Undifferentiated carcinoma of the oropharynx: A human papillomavirus-associated tumor with a favorable prognosis. Mod Pathol 24: 1306-1312, 2011.

2. Lin YC, Wu HP and Tzeng JE: Small-cell undifferentiated carcinoma of the submandibular gland: An extremely rare extrapulmonary site. Am J Otolaryngol 26: 60-63, 2005.

3. Toyosawa S, Ohnishi A, Ito R, Ogawa Y, Kishino M, Yasui Y, Kitamura R, Matsuya T, Ishida T and Ijuhin N: Small cell undifferentiated carcinoma of the submandibular gland: Immunohistochemical evidence of myoepithelial, basal and luminal cell features. Pathol Int 49: 887-892, 1999.

4. Niedobitek G and Herbst H: Epstein-Barr virus-associated carcinomas. Epstein-Barr Virus Rep 1: 81-85, 1994.

5. Ejaz A and Wenig BM: Sinonasal undifferentiated carcinoma: Clinical and pathologic features and a discussion on classification, cellular differentiation, and differential diagnosis. Adv Anat Pathol 12: 134-143, 2005.

6. Ang KK, Harris J, Wheeler R, Weber R, Rosenthal DI, Nguyen-Tân PF, Westra WH, Chung CH, Jordan RC, Lu C, et al: Human papillomavirus and survival of patients with oropharyngeal cancer. N Engl J Med 363: 24-35, 2010.

7. Kofler B, Laban S, Busch CJ, Lörincz B and Knecht R: New treatment strategies for HPV-positive head and neck cancer. Eur Arch Otorhinolaryngol 271: 1861-1867, 2014.

8. Vermorken JB, Mesia R, Rivera F, Remenar E, Kawecki A, Rottey S, Erfan J, Zabolotnyy D, Kienzer HR, Cupissol D, et al: Platinum-based chemotherapy plus cetuximab in head and neck cancer. N Engl J Med 359: 1116-1127, 2008.

9. Iezzoni JC, Gaffey MJ and Weiss LM: The role of Epstein-Barr virus in lymphoepithelioma-like carcinomas. Am J Clin Pathol 103: 308-315, 1995.

10. Wadsworth B, Bumpous JM, Martin AW, Nowacki MR, Jenson $A B$ and Farghaly $\mathrm{H}$ : Expression of p16 in sinonasal undifferentiated carcinoma (SNUC) without associated human papillomavirus (HPV). Head Neck Pathol 5: 349-354, 2011.

11. Okano M, Thiele GM, Davis JR, Grierson HL and Purtilo DT: Epstein-Barr virus and human diseases: Recent advances in diagnosis. Clin Microbiol Rev 1: 300-312, 1988. 
12. Yoshizaki T, Takimoto T, Takeshita H, Tanaka S, Furukawa M, Seiki M and Sato H: Epstein-Barr virus lytic cycle spreads via cell fusion in a nasopharyngeal carcinoma hybrid cell line. Laryngoscope 104: 91-94, 1994.

13. Yoshizaki T, Kondo S, Wakisaka N, Murono S, Endo K, Sugimoto H, Nakanishi S, Tsuji A and Ito M: Pathogenic role of Epstein-Barr virus latent membrane protein-1 in the development of nasopharyngeal carcinoma. Cancer Lett 337: 1-7, 2013

14. Yoshizaki T, Ito M, Murono S, Wakisaka N, Kondo S and Endo K: Current understanding and management of nasopharyngeal carcinoma. Auris Nasus Larynx 39: 137-144, 2012.

15. Kreimer AR, Clifford GM, Boyle P and Franceschi S: Human papillomavirus types in head and neck squamous cell carcinomas worldwide: A systematic review. Cancer Epidemiol Biomarkers Prev 14: 467-475, 2005.
16. Goon PK, Stanley MA, Ebmeyer J, Steinsträsser L, Upile T, Jerjes W, Bernal-Sprekelsen M, Görner M and Sudhoff HH: HPV and head and neck cancer: A descriptive update. Head Neck Oncol 1: 36, 2009.

17. Vermorken JB, Psyrri A, Mesía R, Peyrade F, Beier F, de Blas B, Celik I and Licitra L: Impact of tumor HPV status on outcome in patients with recurrent and/or metastatic squamous cell carcinoma of the head and neck receiving chemotherapy with or without cetuximab: Retrospective analysis of the phase III EXTREME trial. Ann Oncol 25: 801-807, 2014.

18. Bonner JA, Mesia R, Giralt J, Psyrri A, Keilholz U, Rosenthal DI, Beier F, Schulten J and Vermorken JB: p16, HPV, and cetuximab: What is the evidence? Oncologist 22: 811-822, 2017. 